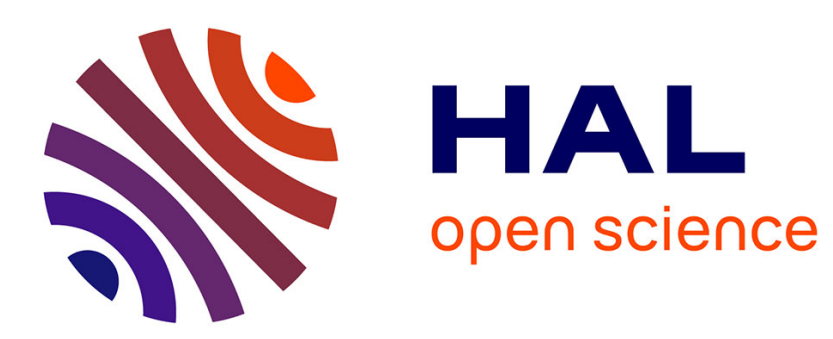

\title{
Design and Transmission Analysis of an Asymmetrical Spherical Parallel Manipulator
}

Guanglei Wu, Stéphane Caro, Jiawei Wang

\section{To cite this version:}

Guanglei Wu, Stéphane Caro, Jiawei Wang. Design and Transmission Analysis of an Asymmetrical Spherical Parallel Manipulator. Mechanism and Machine Theory, 2015, 94, pp.119-131. 10.1016/j.mechmachtheory.2015.07.012 . hal-01757286

\section{HAL Id: hal-01757286 \\ https://hal.science/hal-01757286}

Submitted on 3 Apr 2018

HAL is a multi-disciplinary open access archive for the deposit and dissemination of scientific research documents, whether they are published or not. The documents may come from teaching and research institutions in France or abroad, or from public or private research centers.
L'archive ouverte pluridisciplinaire HAL, est destinée au dépôt et à la diffusion de documents scientifiques de niveau recherche, publiés ou non, émanant des établissements d'enseignement et de recherche français ou étrangers, des laboratoires publics ou privés. 


\title{
Design and Transmission Analysis of an Asymmetrical Spherical Parallel Manipulator
}

\author{
Guanglei Wu${ }^{1 *}$, Stéphane Caro ${ }^{2}$, Jiawei Wang ${ }^{3}$ \\ ${ }^{1}$ Department of Mechanical and Manufacturing Engineering, Aalborg University, 9220 Aalborg, Denmark \\ E-mail:gwu@m-tech.aau.dk \\ ${ }^{2}$ CNRS, Institut de Recherche en Communications et Cybernétique de Nantes \\ UMR CNRS n 6597, 44321 Nantes, France \\ E-mail: Stephane.Caro@irccyn.ec-nantes.fr \\ ${ }^{3}$ Department of Electrical Engineering, Technical University of Denmark, 2800 Kgs. Lyngby, Denmark \\ E-mail:s141378@student.dtu.dk
}

\begin{abstract}
This paper presents an asymmetrical spherical parallel manipulator and its transmissibility analysis. This manipulator contains a center shaft to both generate a decoupled unlimited-torsion motion and support the mobile platform for high positioning accuracy. This work addresses the transmission analysis and optimal design of the proposed manipulator based on its kinematic analysis. The input and output transmission indices of the manipulator are defined for its optimum design based on the virtual coefficient between the transmission wrenches and twist screws. The sets of optimal parameters are identified and the distribution of the transmission index is visualized. Moreover, a comparative study regarding to the performances with the symmetrical spherical parallel manipulators is conducted and the comparison shows the advantages of the proposed manipulator with respect to its spherical parallel manipulator counterparts.
\end{abstract}

Keywords: Asymmetrical spherical parallel manipulator, transmission wrench screw, transmissibility, universal joint

\section{Introduction}

Three degree-of-freedom (3-DOF) spherical parallel manipulators (SPMs) are most widely used as camera-orientating device [1], minimally invasive surgical robots [2] and wrist joints [3] because of their large orientation workspace and high payload capacity. Since they can generate three pure

${ }^{*}$ Corresponding author,Tel:+45 9940 9308; Fax: +45 99407110 
rotations, another potential application is that they can work as a tool head for complicated surface machining. However, the general SPM can only produce a limited torsion motion under a certain tilt orientation, whereas an unlimited torsion is necessary in some common material processing such as milling or drilling. The co-axial input SPM reported in [3] can achieve unlimited torsion, however, its unique structure leads to a complex input mechanism. Moreover, the general SPMs result in low positioning accuracy [4] without a ball-and-socket joint as the center of rotation. In this paper, an asymmetrical SPM (AsySPM) is proposed, which can generate unlimited torsion motion with enhanced positioning accuracy. This manipulator adopts a universal joint as the center of rotation supported by an input shaft at the center, which simplifies the manipulator architecture.

The design of 3-DOF SPMs can be based on many criteria, i.e., workspace [5, 6], dexterity [7, 8, 9], singularity avoidance [10], stiffness [4, 11], dynamics [12, 13], and so on. The prime function of mechanisms is to transmit motion/force between the input joint and the output joint. Henceforth, we will focus on the transmissibility analysis of the proposed SPM. In the design procedure, the performance index is of importance for performance evaluation of the manipulator. A number of transmission indices, such as the transmission angle, the pressure angle, and the transmission factor, have been proposed in the literature to evaluate the quality of motion/force transmission. The transmission angle was introduced by Alt [14], developed by Hain [15], and can be applied in linkage synthesis problems [16, 17]. Takeda et al. [18] proposed a transmission index (TI) for parallel mechanisms based on the minimum value of the cosine of the pressure angle between the leg and the moving platform, where all the inputs but one are fixed. Based on the virtual coefficient between the transmission wrench screw (TWS) and the output twist screw (OTS) introduced by Ball [19], Yuan et al. [20] used it as an unbounded transmission factor for spatial mechanisms. Sutherland and Roth [21] defined the transmission index using a normalized form of the transmission factor, which depends only on the linkages' geometric properties. Chen and Angeles [22] proposed a generalized transmission index that is applicable to single-loop spatial linkages with fixed output and single or multiple DOFs. Wu et al. [23] introduced a frame-free index related to the motion/force transmission analysis for the optimum design of the spherical five-bar mechanism. Wang et al. [24] presented the transmission analysis of fully parallel manipulators based on the transmission indices defined by Sutherland, Roth [21] and Takeda [18]. Recently, some approaches to identify singularity and closeness to singularity have been reported via transmission analysis $[25,26]$. Henceforth, the virtual coefficient based indices will be adopted in this paper for the evaluation of the transmission quality and the optimal design of the proposed manipulator.

This paper presents an asymmetrical SPM and its optimum design with regard to its transmission quality. The inverse and forward kinematic problems of the AsySPM are analyzed based on the kinematic analysis of classical SPMs. By virtue of the virtual coefficient between the transmission wrench screw and output twist screw, the input and output transmission indices are defined for the optimum design, of which an optimization problem is formulated to achieve the optimal design of the proposed SPM. The performances of the proposed spherical manipulator are compared with those of its counterparts in order to highlight its advantages and drawbacks. 


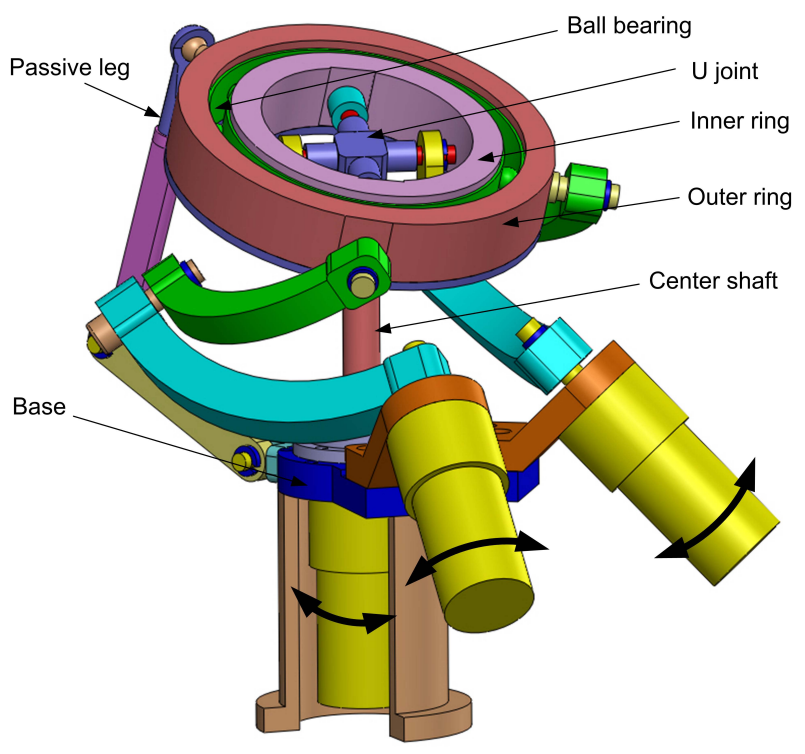

(a)

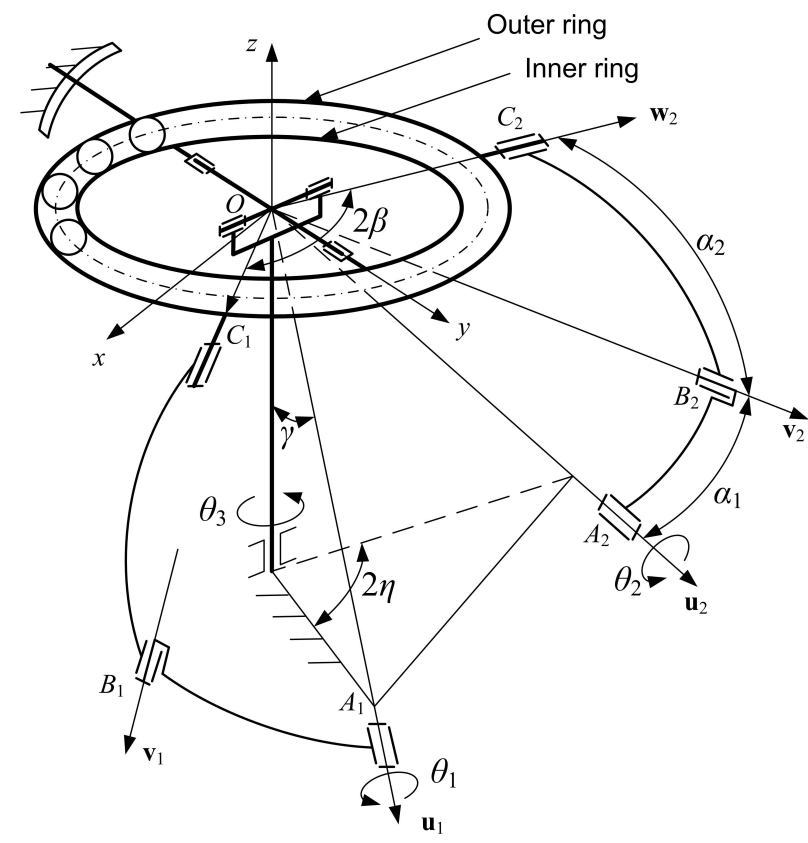

(b)

Figure 1: The asymmetrical spherical parallel manipulator: (a) CAD model; (b) coordinate system.

\section{Kinematics of the Manipulator}

\subsection{Manipulator Architecture}

Figure 1(a) illustrates the asymmetrical SPM, whose mobile platform (MP) is composed of an outer and inner rings connected to each other with a revolute joint, the revolute joint being realized with a revolve bearing. The orientation of the outer ring is determined by two $\underline{\mathrm{RR}^{1}}$ legs and constrained in a vertical plane by a fully passive RRS leg or an alternative RPS one. Through a U-joint, the decoupled rotation of the inner ring is driven by the center shaft, which also supports the MP to improve the positioning accuracy. This manipulator can provide an unlimited rotation of the moving-platform, which can be used in milling or drilling operations and among other material processing. It can also be used as the active spherical joint, i.e., wrist or waist joint.

The coordinate system $(x, y, z)$ is denoted in Fig. 1(b), of which the origin is located at the center of rotation, namely, point $O$. The $i$ th active leg consists of three revolute joints, whose axes are parallel to unit vectors $\mathbf{u}_{i}, \mathbf{v}_{i}, \mathbf{w}_{i}$. Both of these two legs have the same architecture, defined by $\alpha_{1}$ and $\alpha_{2}$ angles. The design parameters of the base platform are $\gamma$ and $\eta$. The design parameter of the mobile platform is $\beta$. It is noteworthy that the manipulator is symmetrical with respect to the $y z$ plane.

\footnotetext{
${ }^{1}$ Throughout this paper, R, U, S and P stand for revolute, universal, spherical and prismatic joints, respectively, and an underlined letter indicates an actuated joint.
} 


\subsection{Inverse Geometric Problem}

Under the prescribed coordinate system, the unit vector $\mathbf{u}_{i}$ is derived as:

$$
\mathbf{u}_{i}=\left[\begin{array}{lll}
(-1)^{i+1} \sin \eta \sin \gamma & \cos \eta \sin \gamma & -\cos \gamma
\end{array}\right]^{T}, \quad i=1,2
$$

The unit vector $\mathbf{v}_{i}$ of the axis of the intermediate revolute joint in the $i$ th leg is obtained in terms of the input joint angle $\theta_{i}$ following the angle-axis representation [27], namely,

$$
\mathbf{v}_{i}=\mathbf{R}\left(\mathbf{u}_{i}, \theta_{i}\right) \mathbf{v}_{i}^{*} ; \quad \mathbf{R}\left(\mathbf{u}_{i}, \theta_{i}\right)=\cos \theta_{i} \mathbf{I}_{3}+\sin \theta_{i}\left[\mathbf{u}_{i}\right]_{\times}+\left(1-\cos \theta_{i}\right) \mathbf{u}_{i} \otimes \mathbf{u}_{i}
$$

where $\mathbf{I}_{3}$ is the identity matrix, $\left[\mathbf{u}_{i}\right]_{\times}$is the cross product matrix of $\mathbf{u}_{i}$ and $\otimes$ is the tensor product. Moreover, $\mathbf{v}_{i}^{*}$ is the unit vector of the axis of the intermediate revolute joint in the $i$ th leg at the original configuration:

$$
\mathbf{v}_{i}^{*}=\left[\begin{array}{lll}
(-1)^{i+1} \sin \eta \sin \left(\gamma+\alpha_{1}\right) & \cos \eta \sin \left(\gamma+\alpha_{1}\right) & -\cos \left(\gamma+\alpha_{1}\right)
\end{array}\right]^{T}
$$

The unit vector $\mathbf{w}_{i}$ of the top revolute joint in the $i$ th leg, is a function of the MP orientation:

$$
\mathbf{w}_{i}=\left[\begin{array}{lll}
x_{i} & y_{i} & z_{i}
\end{array}\right]^{T}=\mathbf{Q} \mathbf{w}_{i}^{*} ; \quad \mathbf{w}_{i}^{*}=\left[\begin{array}{lll}
(-1)^{i+1} \sin \beta & \cos \beta & 0
\end{array}\right]^{T}
$$

where $\mathbf{w}_{i}^{*}$ is the unit vector of the axis of the top revolute joint of the $i$ th leg when the mobile platform is located in its home configuration. Moreover, $\mathbf{Q}=\mathbf{R}\left(x, \phi_{x}\right) \mathbf{R}\left(y, \phi_{y}\right)$ is the rotation matrix of the outer ring. Hence, the orientation of the inner ring can be described with Cardan angles $\left(\phi_{x}, \phi_{y}, \phi_{z}\right)$ and its output axis is denoted by:

$$
\mathbf{p}=\mathbf{Q z} ; \quad \mathbf{z}=[0,0,1]^{T}
$$

According to the motion of the U-joint [28], the input angle $\theta_{3}$ of the center shaft is derived as:

$$
\theta_{3}=\tan ^{-1}\left(\tan \phi_{z} \cos \phi_{x} \cos \phi_{y}\right)
$$

Referring to the inverse kinematic problem of the general SPMs, the loop-closure equation for the $i$ th $\underline{R R R}$ leg is expressed as:

$$
A_{i} t_{i}^{2}+2 B_{i} t_{i}+C_{i}=0, \quad i=1,2
$$

with

$$
\begin{aligned}
& A_{i}=\left((-1)^{i+1} x_{i} \mathrm{~s} \eta+y_{i} \mathrm{c} \eta\right) \mathrm{s}\left(\gamma-\alpha_{1}\right)-z_{i} \mathrm{c}\left(\gamma-\alpha_{1}\right)-\mathrm{c} \alpha_{2} \\
& B_{i}=\left(x_{i} \mathrm{c} \eta-y_{i} \mathrm{~s} \eta\right) \mathrm{s} \alpha_{1} \\
& C_{i}=\left((-1)^{i+1} x_{i} \mathrm{~s} \eta+y_{i} \mathrm{c} \eta\right) \mathrm{s}\left(\gamma+\alpha_{1}\right)-z_{i} \mathrm{c}\left(\gamma+\alpha_{1}\right)-\mathrm{c} \alpha_{2}
\end{aligned}
$$




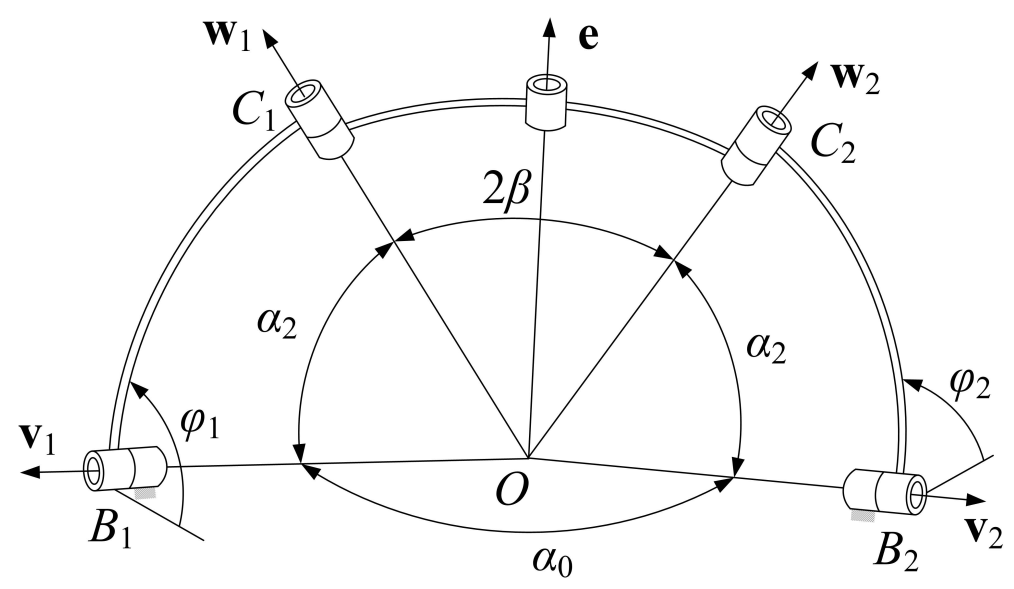

Figure 2: The spherical four-bar linkage.

The input angle displacements can be solved as:

$$
\cos \theta_{i}=\frac{1-t_{i}^{2}}{1+t_{i}^{2}}, \sin \theta_{i}=\frac{2 t_{i}}{1+t_{i}^{2}} ; \quad t_{i}=\tan \frac{\theta_{i}}{2}=\frac{-B_{i} \pm \sqrt{B_{i}^{2}-A_{i} C_{i}}}{A_{i}}
$$

The inverse geometric problem has four solutions corresponding to the four working modes characterized by the sign "-/+" of $\mathbf{u}_{i} \times \mathbf{v}_{i} \cdot \mathbf{w}_{i}$, i.e., "-+", "--", "+-" and "++" modes. Here, the "-+" working mode is selected.

\subsection{Forward Geometric Problem}

The forward geometric problem of the AsySPM can be obtained by searching for the angles $\varphi_{i}$ of a spherical four-bar linkages with the given input angles $\theta_{i}, i=1,2$, as displayed in Fig. 2, where the input/output (I/O) equation takes the form [29, 30]:

$$
f\left(\varphi_{1}, \varphi_{2}\right)=k_{1}+k_{2} \cos \varphi_{1}+k_{3} \cos \varphi_{1} \cos \varphi_{2}-k_{4} \cos \varphi_{2}+k_{5} \sin \varphi_{1} \sin \varphi_{2}=0
$$

with

$$
k_{1} \equiv \mathrm{C} \alpha_{0} \mathrm{C}^{2} \alpha_{2}-\mathrm{C} \beta^{\prime} ; \quad k_{2}=k_{4} \equiv \mathrm{S} \alpha_{0} \mathrm{~S} \alpha_{2} \mathrm{C} \alpha_{2} ; \quad k_{3} \equiv \mathrm{C} \alpha_{0} \mathrm{~S}^{2} \alpha_{2} ; \quad k_{5} \equiv \mathrm{S}^{2} \alpha_{2}
$$

where $\mathrm{S}$ and $\mathrm{C}$ stand for the sine and cosine functions, respectively, and $\alpha_{0}=\cos ^{-1}\left(\mathbf{v}_{1} \cdot \mathbf{v}_{2}\right), \beta^{\prime}=2 \beta$.

On the other hand, the motion of the unit vector $\mathbf{e}$ is constrained in the $y z$ plane due to the passive leg, thus:

$$
g\left(\varphi_{1}, \varphi_{2}\right)=x_{1}+x_{2}=0
$$

where the unit vector $\mathbf{w}_{i}$ can be also represented with angle-axis rotation matrix, namely,

$$
\mathbf{w}_{i}=\left[\begin{array}{lll}
x_{i} & y_{i} & z_{i}
\end{array}\right]^{T}=\mathbf{R}\left(\mathbf{v}_{i}, \varphi_{i}\right) \mathbf{R}\left(\mathbf{v}_{0}, \alpha_{2}\right) \mathbf{v}_{i} ; \quad \mathbf{v}_{0}=\mathbf{v}_{1} \times \mathbf{v}_{2} /\left\|\mathbf{v}_{1} \times \mathbf{v}_{2}\right\|
$$




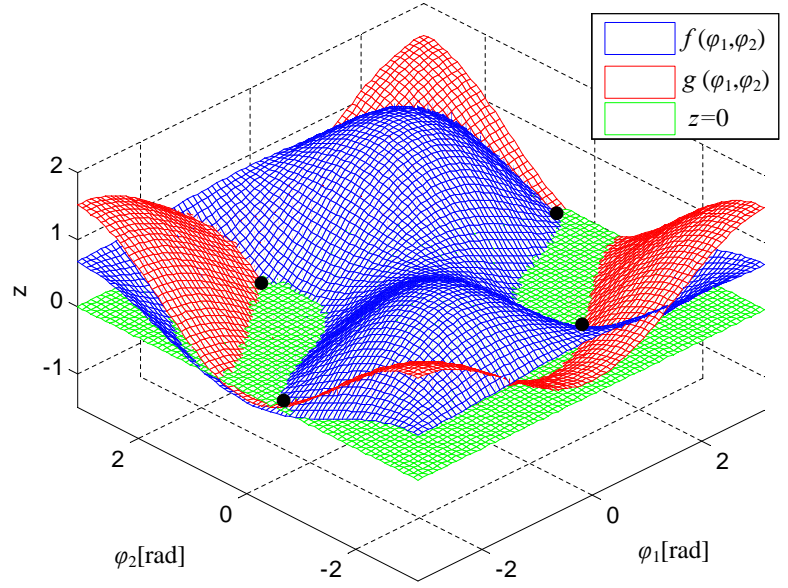

Figure 3: Graphical representation (four black points) of the four solutions to the forward geometric problem of the AsySPM.

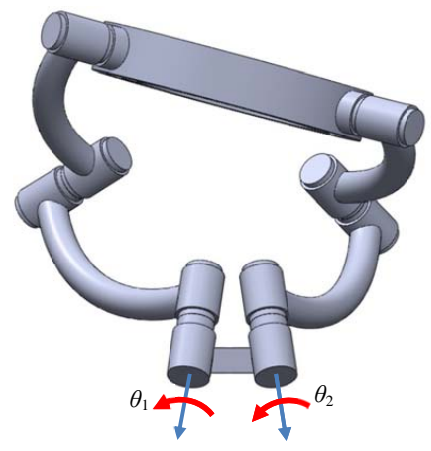

(a)

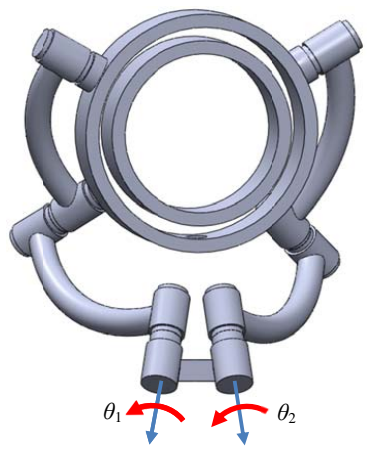

(b)

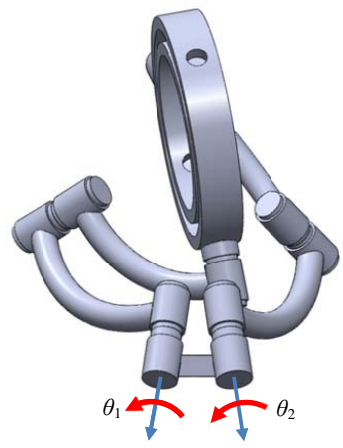

(c)

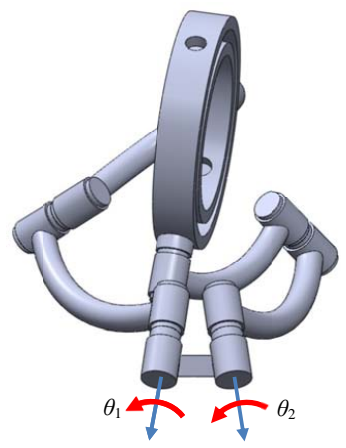

(d)

Figure 4: The four assembly modes of the AsySPM.

Solving Eqs. (10) and (12) leads to four solutions for the angles $\varphi_{i}, i=1$, 2, i.e., the two functions have four common points in the plane $z=0$ as shown in Fig. 3. Figure 4 illustrates the four assembly modes corresponding to the four solutions. Then, substituting $\varphi_{i}$ into Eq. (13), the unit vector $\mathbf{w}_{i}$ and the output Euler angles $\phi_{x}$ and $\phi_{y}$ can be obtained, and the output angle $\phi_{z}$ can be obtained from Eq. (6) accordingly.

\section{Transmission Index}

The main function of the mechanism is to transmit motion from the input element to the output element. As a result, the force applied to the output element is to be transmitted to the input one. The arising internal wrench due to transmission is defined as a transmission wrench, which is characterized by the magnitude of the force and transmission wrench screw (TWS), and the latter is used to evaluate the quality of the transmission. In order to evaluate the transmission performance of the manipulator, some transmission indices (TI) should be defined. 


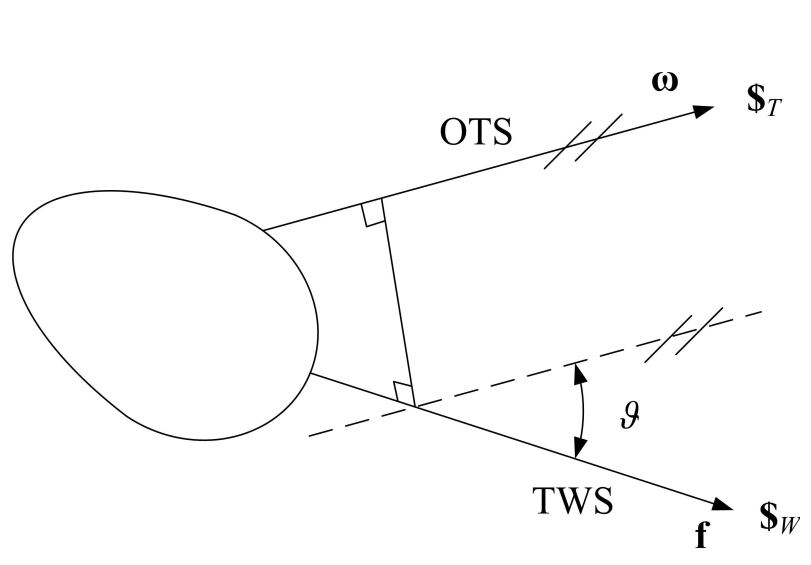

(a)

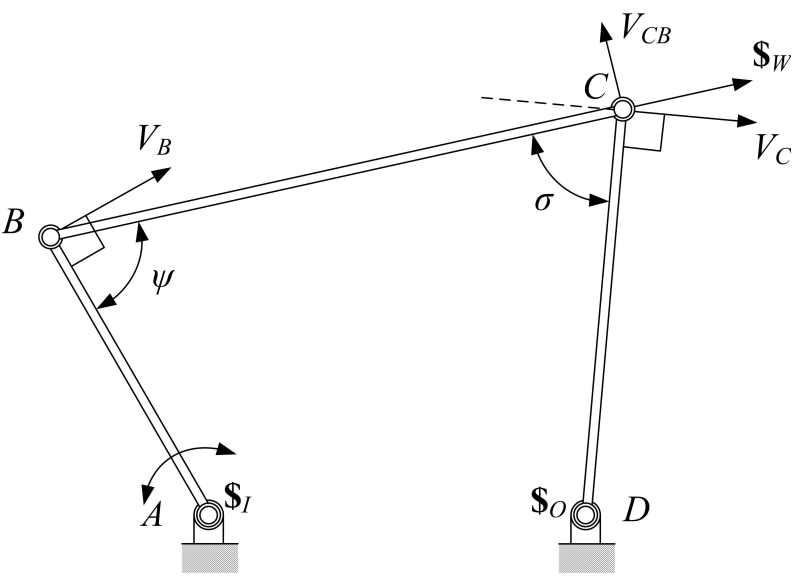

(b)

Figure 5: Transmission wrench and twist screw: (a) the twist screw and wrench screw of a rigid body; (b) a planar four-bar linkage.

\subsection{Transmission Wrench and Twist Screw}

As shown in Fig. 5(a), the instantaneous motion of a rigid body can be represented by using a twist screw defined by its Plücker coordinates:

$$
\$_{T}=(\boldsymbol{\omega} ; \mathbf{v})=\omega \hat{\boldsymbol{\$}}_{T}=\left(L_{1}, M_{1}, N_{1} ; P_{1}^{*}, Q_{1}^{*}, R_{1}^{*}\right)
$$

where $\omega$ is the amplitude of the twist screw and $\hat{\$}_{T}$ is the unit twist screw. Likewise, a wrench exerted on the rigid body can be expressed as a wrench screw defined by its Plücker coordinates as:

$$
\$_{W}=(\mathbf{f} ; \mathbf{m})=f \hat{\$}_{W}=\left(L_{2}, M_{2}, N_{2} ; P_{2}^{*}, Q_{2}^{*}, R_{2}^{*}\right)
$$

where $f$ is the amplitude of the wrench screw and $\hat{\$}_{W}$ is the unit wrench screw.

The reciprocal product between the two screws $\$_{T}$ and $\$_{W}$ is defined as:

$$
\$_{T} \circ \$_{W}=\mathbf{f} \cdot \mathbf{v}+\mathbf{m} \cdot \boldsymbol{\omega}=L_{1} P_{2}^{*}+M_{1} Q_{2}^{*}+N_{1} R_{2}^{*}+L_{2} P_{1}^{*}+M_{2} Q_{1}^{*}+N_{2} R_{1}^{*}
$$

This reciprocal product amounts to the instantaneous power between the wrench and the twist. Subsequently, the transmission index is defined as a dimensionless index [22]:

$$
\mathrm{TI}=\frac{\left|\hat{\boldsymbol{\$}}_{T} \circ \hat{\boldsymbol{\$}}_{W}\right|}{\left|\hat{\boldsymbol{\$}}_{T} \circ \hat{\boldsymbol{\$}}_{W}\right|_{\max }}
$$

where $\left|\hat{\$}_{T} \circ \hat{\$}_{W}\right|_{\max }$ represents the potential maximal magnitude of the reciprocal product between $\hat{\boldsymbol{\$}}_{T}$ and $\hat{\boldsymbol{\$}}_{W}$. The larger TI, the more important the power transmission from the wrench to the twist, namely, the better the transmission quality.

For a planar manipulator, this index corresponds to the transmission angle, which is the smallest 


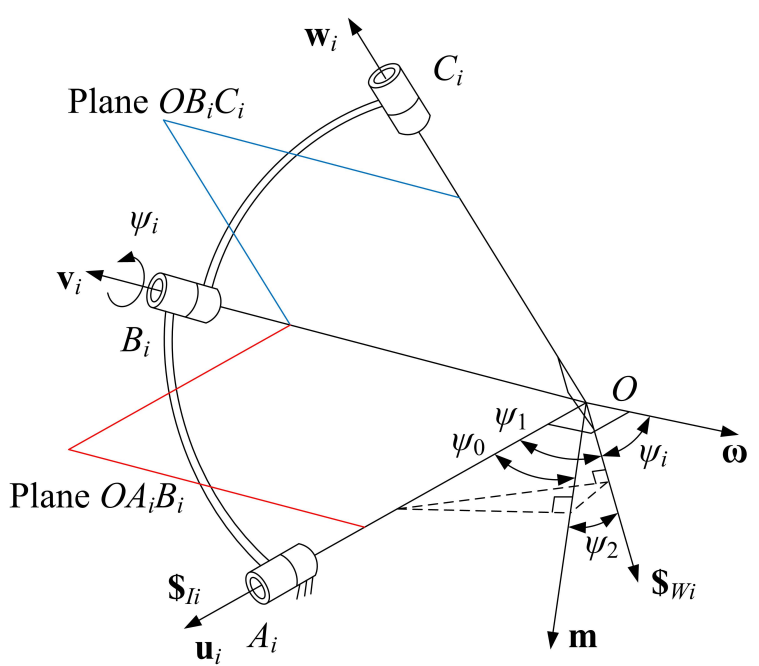

(a)

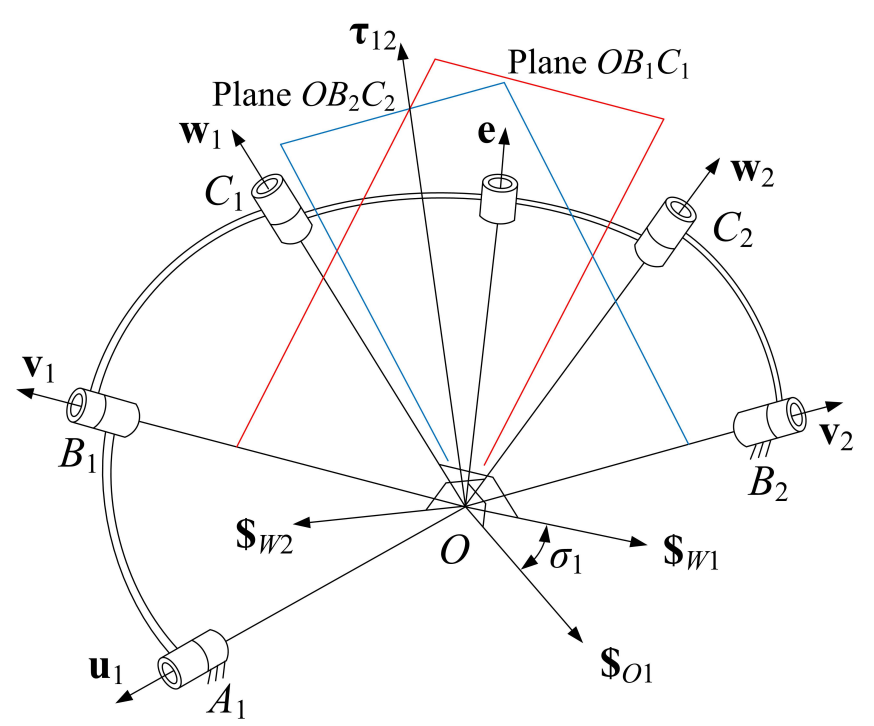

(b)

Figure 6: The transmission wrench screw and transmission angle: (a) input transmission; (b) output tranmission.

angle between the direction of velocity of the driven link and the direction of absolute velocity vector of output link both taken at the common point [31]. As illustrated in Fig. 5(b), it is the angle $\sigma$ between the follower link and the coupler of a four-bar mechanism, also known as forward transmission angle. Conversely, the angle $\psi$ is the inverse transmission angle. Therefore, the input $\left(\lambda_{I}\right)$ and output $\left(\lambda_{O}\right)$ transmission can be expressed as:

$$
\lambda_{I}=|\sin \psi| \quad ; \quad \lambda_{O}=|\sin \sigma|
$$

\subsection{Input Transmission Index}

The wrench applied to a SPM is usually a pure moment, thus, for a spherical $\underline{R R R ~ l e g, ~ t h e ~ t r a n s m i s s i o n ~}$ wrench is a pure torque. As the TWS is reciprocal to all the passive joint screws in the leg, the axis of the wrench in the $i$ th leg is perpendicular to the plane $O B_{i} C_{i}$ and passes through point $O$, as shown in Fig. 6(a). According to Eq. (17), the input transmission index of the $i$ th RRR leg is obtained as:

$$
\lambda_{I i}=\frac{\left|\hat{\$}_{I i} \circ \hat{\$}_{W i}\right|}{\left|\hat{\$}_{I i} \circ \hat{\$}_{W i}\right|_{\max }}=\frac{\left|\mathbf{u}_{i} \cdot \boldsymbol{\tau}_{i}\right|}{\left|\mathbf{u}_{i} \cdot \boldsymbol{\tau}_{i}\right|_{\max }}, \quad i=1,2
$$

with

$$
\hat{\$}_{I i}=\left(\mathbf{u}_{i} ; \mathbf{0}\right) ; \quad \hat{\$}_{W i}=\left(\mathbf{0} ; \boldsymbol{\tau}_{i}\right)=\left(\mathbf{0} ; \mathbf{v}_{i} \times \mathbf{w}_{i} /\left\|\mathbf{v}_{i} \times \mathbf{w}_{i}\right\|\right)
$$

When $\hat{\$}_{W i}$ lies in the plane $O A_{i} B_{i}$, i.e., plane $O A_{i} B_{i}$ being perpendicular to plane $O B_{i} C_{i},\left|\mathbf{u}_{i} \cdot \boldsymbol{\tau}_{i}\right|$ reaches its maximum value. This situation occurs when the angle between the wrench screw and the 
twist screw is equal to $\psi_{0}$ or $180^{\circ}-\psi_{0}$, namely,

$$
\left|\mathbf{u}_{i} \cdot \boldsymbol{\tau}_{i}\right|_{\max }=\left|\cos \psi_{0}\right|=\left|\sin \alpha_{1}\right|
$$

From Fig. 6(a), Eq. (19) is equivalent to

$$
\lambda_{I i}=\frac{\left|\cos \psi_{1}\right|}{\left|\cos \psi_{0}\right|}=\left|\cos \psi_{2}\right|=\left|\sin \psi_{i}\right|=\sqrt{1-\cos ^{2} \psi_{i}}, i=1,2
$$

where $\psi_{i}$ is the inverse transmission angle, i.e., the angle between planes $O A_{i} B_{i}$ and $O B_{i} C_{i}$, and

$$
\cos \psi_{i}=\frac{\left(\mathbf{v}_{i} \times \mathbf{u}_{i}\right) \cdot\left(\mathbf{v}_{i} \times \mathbf{w}_{i}\right)}{\left\|\mathbf{v}_{i} \times \mathbf{u}_{i}\right\|\left\|\mathbf{v}_{i} \times \mathbf{w}_{i}\right\|}
$$

Finally, the input transmission index of the manipulator is defined as:

$$
\lambda_{I}=\min \left\{\lambda_{I i}\right\}, \quad i=1,2
$$

\subsection{Output Transmission Index}

Referring to the pressure angle at the attached point of the leg with the moving platform [18], the output transmission index of a single leg can be defined by fixing the other input joints, where the parallel manipulator thus becoming a 1-DOF system. By fixing the active joint at point $A_{2}$ (point $B_{2}$ will be fixed) and keeping joint at point $A_{1}$ actuated in Fig. 6(b), the transmission wrench $\$_{W 2}$ becomes a constraint wrench for the mobile platform. The instantaneous motion of the mobile platform will be a rotation about a unique vector constrained by $\$_{W 2}$ and the vector e in the passive leg, namely,

$$
\mathbf{s}_{1}=\frac{\boldsymbol{\tau}_{2} \times \mathbf{e}}{\left\|\boldsymbol{\tau}_{2} \times \mathbf{e}\right\|} ; \quad \mathbf{e}=\mathbf{Q j}, \mathbf{j}=[0,1,0]^{T}
$$

Henceforth, the output twist screw can be expressed as: $\hat{\boldsymbol{\$}}_{O 1}=\left(\mathbf{s}_{1} ; \mathbf{0}\right)$. Based on Eq. (17), the output transmission index of the first leg is defined by

$$
\lambda_{O 1}=\frac{\left|\hat{\$}_{O 1} \circ \hat{\$}_{W 1}\right|}{\left|\hat{\$}_{O 1} \circ \hat{\$}_{W 1}\right|_{\max }}=\frac{\left|\mathbf{s}_{1} \cdot \boldsymbol{\tau}_{1}\right|}{\left|\mathbf{s}_{1} \cdot \tau_{1}\right|_{\max }}
$$

when $\mathbf{s}_{1}$ and $\boldsymbol{\tau}_{1}$ are parallel, i.e., both planes $O C_{1} C_{2}$ and $O B_{1} C_{1}$ being perpendicular to $O B_{2} C_{2}$, $\left|\mathbf{s}_{1} \cdot \boldsymbol{\tau}_{1}\right|$ is a maximum, namely, $\left|\mathbf{s}_{1} \cdot \boldsymbol{\tau}_{1}\right|_{\max }=\cos (0)=1$. Equation $(26)$ is rewritten as:

$$
\lambda_{O 1}=\left|\mathbf{s}_{1} \cdot \boldsymbol{\tau}_{1}\right|=\left|\boldsymbol{\tau}_{12} \cdot \mathbf{e}\right| /\left\|\boldsymbol{\tau}_{2} \times \mathbf{e}\right\| ; \quad \boldsymbol{\tau}_{12}=\tau_{1} \times \boldsymbol{\tau}_{2}
$$

By the same token, the output transmission index of the second leg is derived as:

$$
\lambda_{O 2}=\left|\boldsymbol{\tau}_{12} \cdot \mathbf{e}\right| /\left\|\boldsymbol{\tau}_{1} \times \mathbf{e}\right\|
$$


Similarly to Eq. (24), the output transmission index of the manipulator is defined as

$$
\lambda_{O}=\min \left\{\lambda_{O i}\right\}, \quad i=1,2
$$

\subsection{Transmission Efficiency of the $U$ joint}

The output of the inner ring of the mobile platform is driven by the center shaft through a universal joint, consequently, the TI of the U joint (UTI) is defined as:

$$
\lambda_{U}=|\mathbf{p} \cdot \mathbf{z}|=\left|\cos \phi_{x} \cos \phi_{y}\right|
$$

where the vectors $\mathbf{p}$ and $\mathbf{z}$ are defined in Eq. (5).

\subsection{Local Transmission Index}

On the basis of the ITI, OTI and UTI, the local transmission index (LTI) of the manipulator under study, namely, the transmission index at a prescribed orientation, is defined as:

$$
\lambda=\min \left\{\lambda_{I}, \lambda_{O}, \lambda_{U}\right\}
$$

The higher $\lambda$, the higher the quality of the input and output transmission. The distribution of the LTI can indicate the workspace (WS) region with a good transmissibility. Thus, this index can be used for either the evaluation of the transmission quality or the design optimization.

\section{Optimal Design and Analysis}

The optimum design of SPMs can be based on many aspects, such as workspace, dexterity, singularity, and so on. However, these criteria are usually antagonistic. In order for the proposed AsySPM to achieve a regular workspace (RWS) with a good transmission quality, the following optimization problem is formulated:

$$
\begin{aligned}
\operatorname{maximize} & f(\mathbf{x})=\lambda \quad \text { for } \quad \theta \in\left[0, \theta_{0}\right] \\
\text { over } & \mathbf{x}=\left[\alpha_{1} ; \alpha_{2} ; \beta ; \gamma ; \eta\right] \\
\text { subject to } & g_{1}: 45^{\circ} \leq\left\{\alpha_{1}, \alpha_{2}\right\} \leq 120^{\circ} \\
& g_{2}: 15^{\circ} \leq\{\beta, \eta\} \leq 60^{\circ} \\
& g_{3}: 30^{\circ} \leq \gamma \leq 75^{\circ}
\end{aligned}
$$

where $\theta$ is the tilt angle and $\theta_{0}$ defines the workspace region, as shown in Fig. 7. Moreover, the lower and upper bounds of the design variables are assigned in order to avoid mechanical collisions. This problem can be solved with the optimization toolbox of the mathematical software at hand. Hereby, it is solved with the genetic algorithm (GA) toolbox in Matlab. When $\theta_{0}=60^{\circ}$, it is found that 


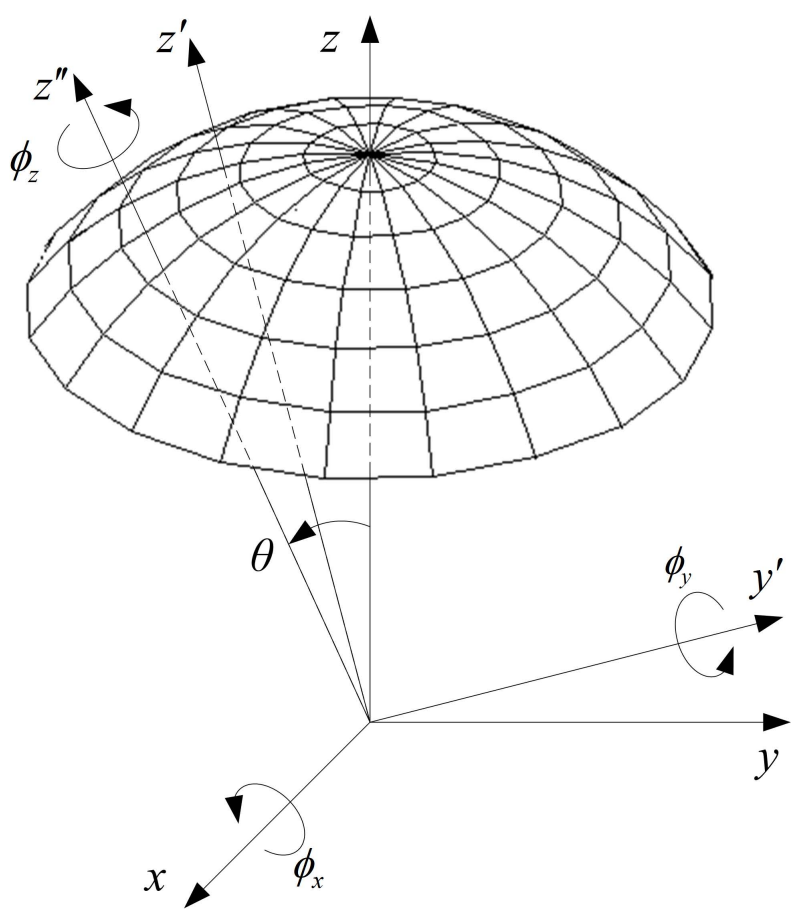

Figure 7: The spherical surface of a designated regular workspace.

the solution of optimization problem (32) leads to different local optimums, which implies that the minimum LTI depends on the I/O angle of the universal joint when the geometrical parameters subject to some certain ranges, and for instance, one solution is found as:

$$
\mathbf{x}=\left[60^{\circ} ; 75^{\circ} ; 15^{\circ} ; 60^{\circ} ; 60^{\circ}\right]
$$

The corresponding LTI is shown in Fig. 8(a), where the solid black circle stands for the reachable WS boundary of $\theta=75^{\circ}$ and the minimum LTI is equal to 0.5 with $\theta \in\left[0,60^{\circ}\right]$. In the literature, the recommended transmission angle is $90^{\circ} \pm 50^{\circ}[14]$ and the most widely accepted is $\left[45^{\circ}, 135^{\circ}\right]$ for highquality transmission [32], whence the TI is $\lambda=\sin 45^{\circ} \approx 0.7$, i.e., the manipulator at a configuration with LTI $\lambda \geq 0.7$ has good motion/force transmission. Henceforth, a set of poses in which LTI is greater than 0.7 is identified as high-transmissibility workspace (HTW), such as the blue dashed line enveloped region displayed in Fig. 8(a). The area of HTW can be used to evaluate the manipulator performance. The larger the HTW, the better the transmission quality of the manipulator.

When the objective function in the optimization problem (32) is replaced by $f(\mathbf{x})=A_{\mathrm{HTW}}$, where $A_{\mathrm{HTW}}$ is the area of HTW, the optimal parameters for $\theta_{0}=60^{\circ}$ are found as:

$$
\mathbf{x}=\left[53.3^{\mathrm{o}} ; 69.5^{\mathrm{o}} ; 15^{\mathrm{o}} ; 75^{\mathrm{o}} ; 60^{\circ}\right]
$$

The corresponding distribution of performance index is shown in Fig. 8(b), from which it is seen that the manipulator can still reach a large RWS with $\theta=75^{\circ}$, whereas, the minimum LTI within $\theta \in\left[0,60^{\circ}\right]$ reduces to 0.3 compared to Fig. 8(a). In contrast, the HTW of the manipulator with the 


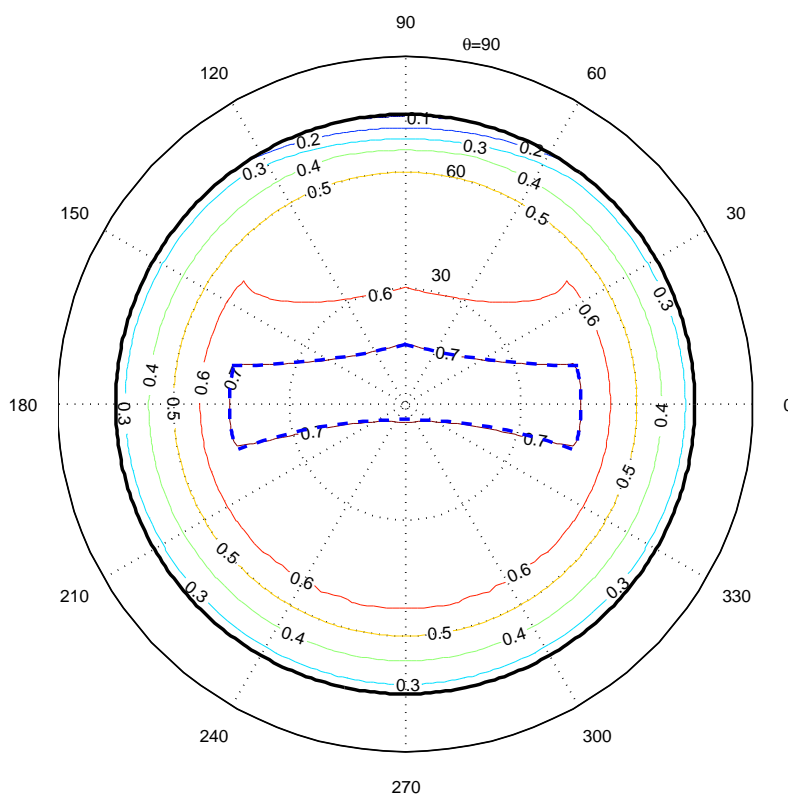

(a)

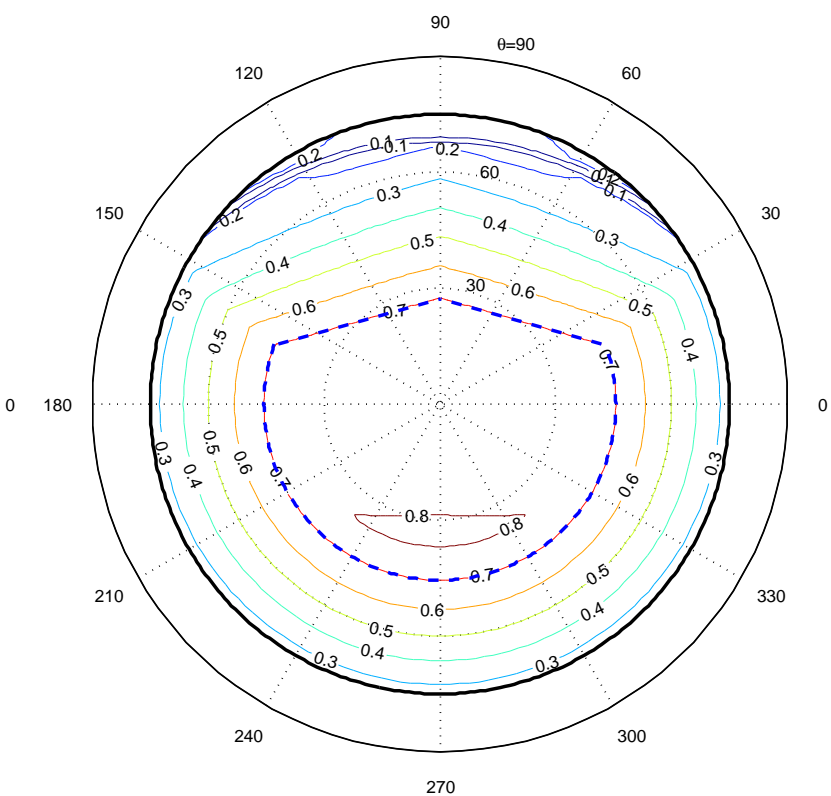

(b)

Figure 8: The isocontours of the transmission index of the asymmetrical SPM for workspace $\theta_{0}=60^{\circ}$ : (a) $\max (\lambda)$; (b) $\max (\mathrm{HTW})$.

geometrical parameters given in Eq. (34) is much larger than the HTW of the manipulator with the geometrical parameters given in Eq. (33).

To evaluate the transmissibility of the manipulator within a designated workspace, a transmission index (WTI) similar to GCI [7] is defined over the workspace $W$, which is calculated through a discrete approach in practice, namely,

$$
\mathrm{WTI}=\frac{\int \lambda \mathrm{d} W}{\int \mathrm{d} W} \quad \text { or } \quad \mathrm{WTI}=\frac{1}{W} \sum_{i=1}^{n} \lambda_{i} \Delta W=\frac{1}{n} \sum_{i=1}^{n} \lambda_{i}
$$

where $n$ is the discrete number. The index obtained through the above equation is an arithmetic mean, which can be replaced with a quadratic mean for a better indication of the transmission, subsequently, WTI is redefined as

$$
\mathrm{WTI}=\sqrt{\frac{1}{n} \sum_{i=1}^{n} \lambda_{i}^{2}}
$$

As a consequence, with $\theta \in\left[0,60^{\circ}\right]$, WTI is equal to 0.66 for the first design and is equal to 0.72 for the second one.

\section{Comparison with Symmetrical SPMs}

In this section, a comparative study is conducted between the asymmetrical and symmetrical SPMs. A general SPM is shown in Fig. 9(a), which consists of three identical $\underline{R} R R$ legs connected to the base and the mobile platform. Moreover, $\beta$ and $\gamma$ define the geometry of two triangular pyramids on the 


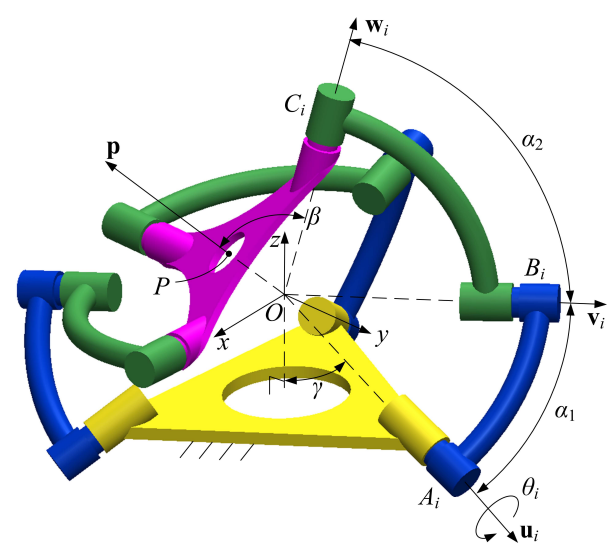

(a)

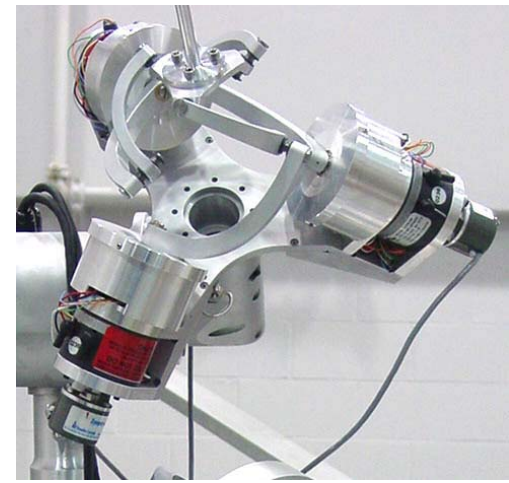

(b)

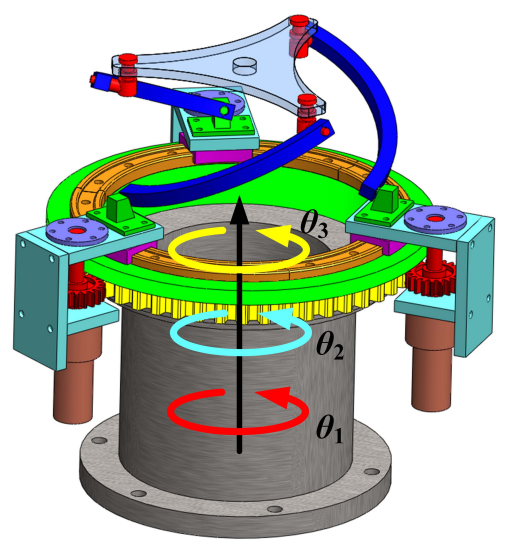

(c)

Figure 9: The symmetrical SPMs: (a) general SPM; (b) Agile Wrist; (c) co-axial input SPM.

Table 1: Geometrical parameters of the Agile Wrist and CoSPM.

\begin{tabular}{llllll}
\hline \hline \multicolumn{2}{c}{ Agile Wrist } & & \multicolumn{3}{c}{ CoSPM } \\
\cline { 1 - 1 } \cline { 5 - 6 }$\alpha_{1}, \alpha_{2}[\mathrm{deg}]$ & $\beta, \gamma[\mathrm{rad}]$ & & $\alpha_{1}[\mathrm{deg}]$ & $\alpha_{2}[\mathrm{deg}]$ & $\beta[\mathrm{deg}]$ \\
\hline 90 & $\sin ^{-1}(\sqrt{6} / 3)$ & & $60(47)$ & 90 & 90 \\
\hline \hline
\end{tabular}

mobile and the base platforms, respectively. A base coordinate system $(x, y, z)$ is located at point $O$ and the $z$ axis is normal to the bottom surface of the base pyramid and points upwards, while the $y$ axis is located in the plane spanned by the $z$-axis and vector $\mathbf{u}_{1}$. Figure $9(\mathrm{~b})$ illustrates the Agile Wrist [11] while Fig. 9(c) shows a co-axial input SPM (CoSPM) of a special case with $\gamma=0$. Their geometrical parameters are given by Table 1 .

\subsection{LTI distributions}

Referring to Eqs. (19) and (26), the ITI and OTI of each leg for the symmetrical SPMs can be obtained. The difference lies in the output twist screw of Eq. (25) in the calculation of OTI, namely,

$$
\mathbf{s}_{i}=\frac{\left(\mathbf{v}_{j} \times \mathbf{w}_{j}\right) \times\left(\mathbf{v}_{k} \times \mathbf{w}_{k}\right)}{\left\|\left(\mathbf{v}_{j} \times \mathbf{w}_{j}\right) \times\left(\mathbf{v}_{k} \times \mathbf{w}_{k}\right)\right\|} ; \quad i, j, k \in\{1,2,3\}, i \neq j \neq k
$$

Using the index defined in Eq. (31), the LTI distributions of the Agile Wrist and CoSPM are shown in Figs. 10 and 11, respectively.

The Agile Wrist has a large WS with limited torsion angles, as shown in Fig. 10. The LTI within the workspace $\theta \in\left[0,60^{\circ}\right]$ is bounded between 0.2 and 0.5 . Its HTW becomes larger with the increasing torsion angle, however, as displayed in Fig. 10(a), the HTW with $\phi_{z} \in\left[-30^{\circ}, 30^{\circ}\right]$ is much smaller than that of the AsySPM. The WTI with the range $\phi_{z} \in\left[-30^{\circ}, 30^{\circ}\right]$ of the torsion angle is shown in Table 2 with the comparison to other SPMs.

The CoSPM has a relatively small reachable WS as shown in Fig. 11, i.e., the region enveloped 


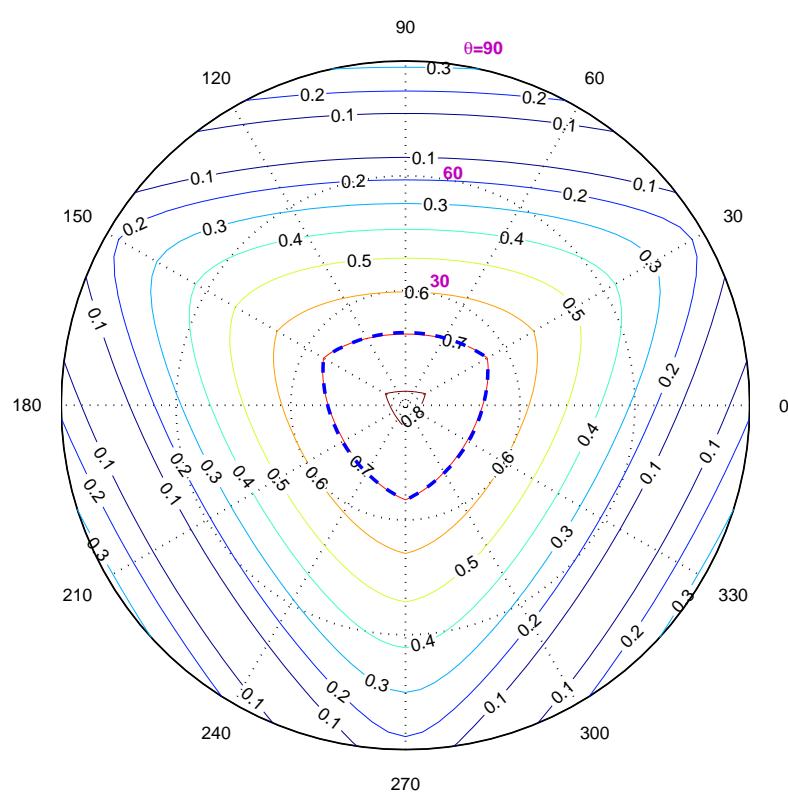

(a)

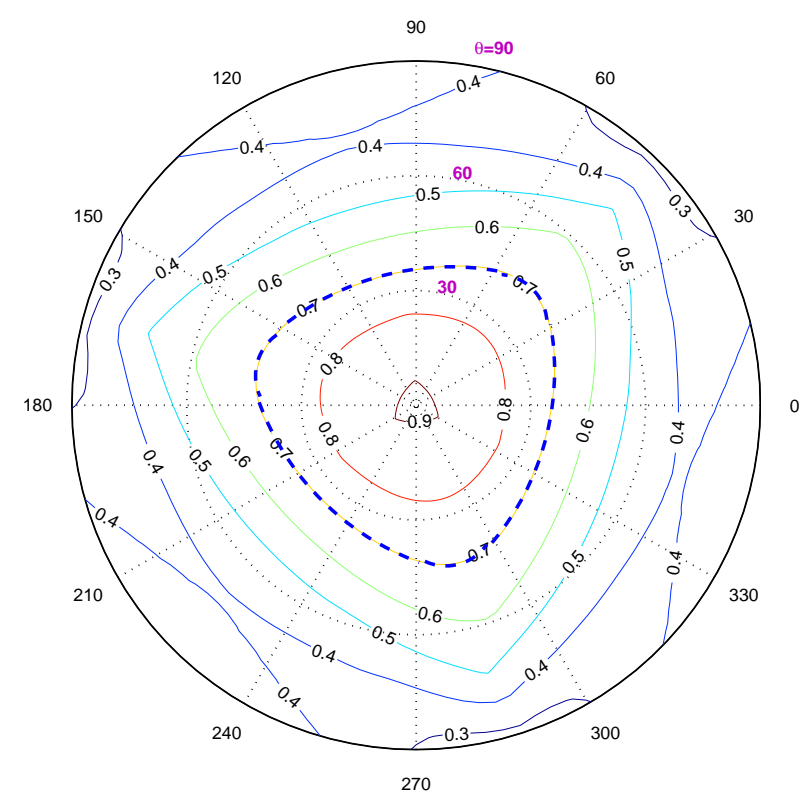

(b)

Figure 10: The LTI isocontours of the Agile Wrist: (a) $\phi_{z}=0$; (b) $\phi_{z}=30^{\circ}$.

Table 2: WTI for the three SPMs.

\begin{tabular}{clllll}
\hline \hline & \multicolumn{2}{c}{ AsySPM } & Agile Wirst & \multicolumn{2}{c}{ CoSPM } \\
\cline { 2 - 3 } \cline { 5 - 6 } & parameters (33) & parameters (34) & & $\alpha_{1}=60^{\circ}$ & $\alpha_{1}=47^{\circ}$ \\
\hline$\theta \in\left[0,45^{\circ}\right]$ & 0.68 & 0.77 & 0.75 & 0.58 & 0.79 \\
$\theta \in\left[0,60^{\circ}\right]$ & 0.66 & 0.72 & 0.69 & 0.54 & \\
\hline \hline
\end{tabular}




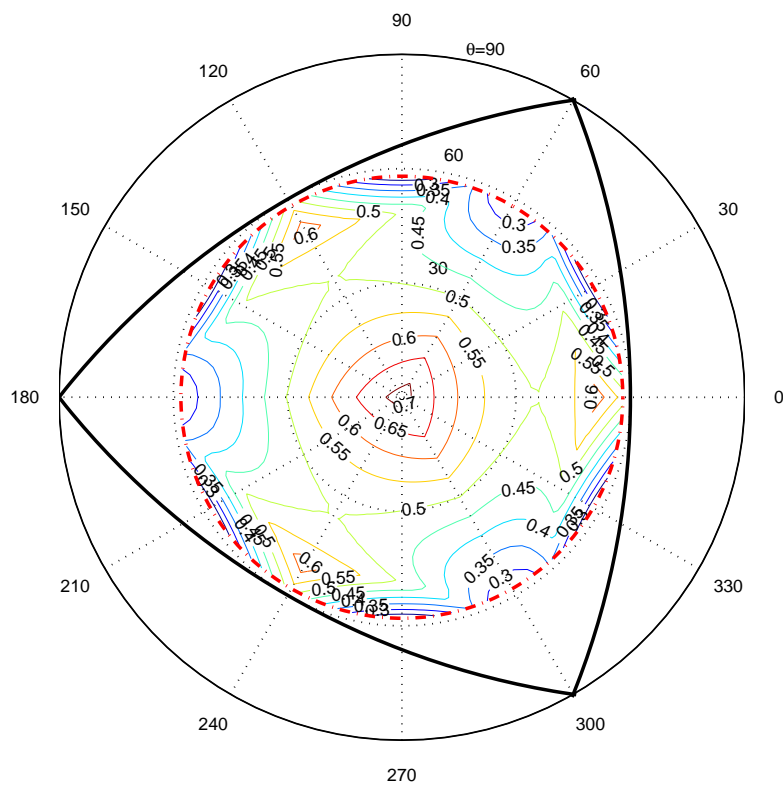

(a)

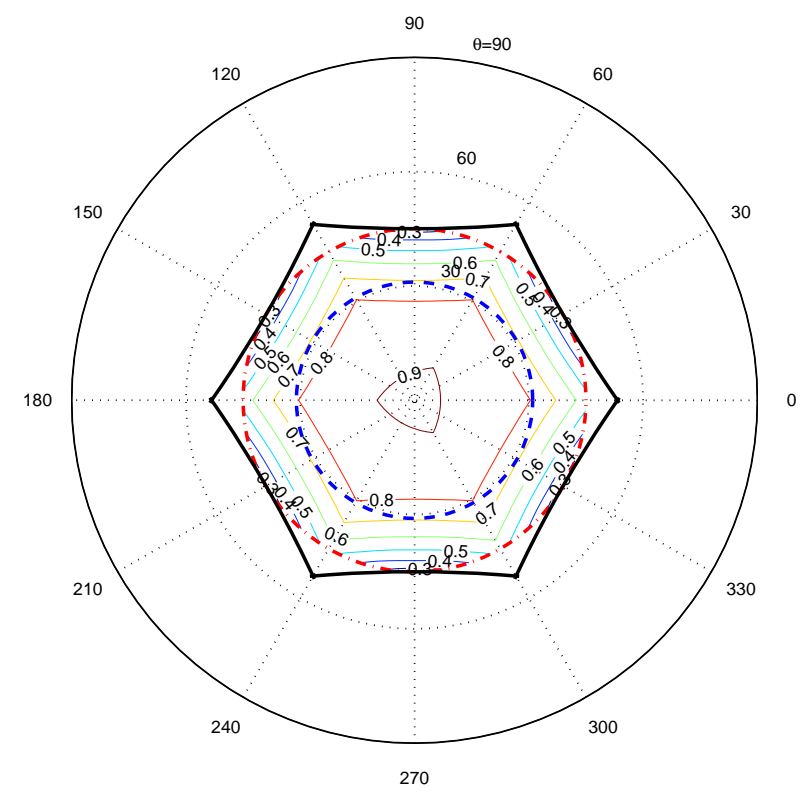

(b)

Figure 11: The LTI isocontours of the CoSPM with torsion $\phi_{z}=0$ : (a) $\alpha_{1}=60^{\circ}$; (b) $\alpha_{1}=47^{\circ}$.

by the solid black arcs. The LTI distributions with torsion angle $\phi_{z}=0$ for two different designs are displayed and the index maps with different torsion angles are rotationally symmetrical to them. It is found that the minimum transmission indices over the regular workspace for these two designs are both equal to 0.3. Although the proximal link $\alpha_{1}=60^{\circ}$ admits a relatively large dexterous WS [6], the HTW is extremely small. When $\alpha_{1}$ reduces to $47^{\circ}$ which yields better kinematic and dynamic dexterities [13], the HTW of full torsion is a spherical cap with $\theta \in\left[0,30^{\circ}\right]$.

\subsection{Comparison of Overall Performances}

The performance comparison of the asymmetrical SPM with the Agile Wrist and the Co-axial input spherical parallel manipulator is summarized in Table 3, which shows the advantages and drawbacks of the proposed AsySPM with respect to its symmetrical counterparts. The AsySPM can have the advantages of the general and co-axial input SPMs simultaneously except the drawback of evenly distributed power consumption.

\section{Conclusion}

This paper introduced an asymmetrical spherical parallel manipulator, whose the mobile platform is composed of an inner ring and an outer ring. The orientation of the outer ring is determined by two $\underline{R R R}$ legs as well as a fully passive leg, and the inner ring can generate a decoupled unlimited-torsion motion thanks to a center input shaft and a universal joint. Moreover, the center shaft can improve the positioning accuracy of the center of rotation for the manipulator. This manipulator can be used as a tool head for the complicated surface machining, such as milling or drilling, and can also work as 
Table 3: Performance comparison of the asymmetrical SPM with the Agile Wrist and the Co-axial input SPM.

\begin{tabular}{llll}
\hline \hline & AsySPM & Agile Wrist & CoSPM \\
\hline Compactness & + & + & \\
Low inertia/mass & + & + & \\
Regular WS & + & + & \\
Decoupled mobility & + & & \\
Unlimited torsion motion & + & & + \\
Positioning accuracy (center shift $[4])$ & ++ & & + \\
Even power consumption & + & ++ & ++ \\
HTW, WTI & ++ & + & + \\
\hline \hline
\end{tabular}

an active spherical joint.

As one of the most important performance, transmission analysis for the proposed manipulator was addressed. By virtue of the transmission wrench screw and the output twist screw, the input and output transmission indices are defined and are further used for the optimal design of the proposed manipulator. Two sets of optimal parameters have been identified and the isocontours of the transmission indices were traced to show the quality of the transmission. Moreover, a comparative study dealing with the mechanical design, kinematic and transmission performances was carried out between the proposed manipulator and its symmetrical counterparts, which highlights the advantages and drawbacks of the proposed manipulator with respect to its symmetrical counterparts. Besides the advantages of the general spherical parallel manipulators, such as compactness, low inertia, large regular workspace, the proposed manipulator can outperform in terms of unlimited torsion rotation, positioning accuracy and transmission quality, on the other hand, a main drawback lies in the unevenly distributed power consumption due to its asymmetrical structure.

\section{References}

[1] C.M. Gosselin and J.F. Hamel. The Agile Eye: a high-performance three-degree-of-freedom camera-orienting device. In IEEE International Conference on Robotics and Automation, pages 781-786, 1994.

[2] T. Li and S. Payandeh. Design of spherical parallel mechanisms for application to laparoscopic surgery. Robotica, 20(2):133-138, 2002.

[3] H. Asada and J. Granito. Kinematic and static characterization of wrist joints and their optimal design. In IEEE International Conference on Robotics and Automation, pages 244-250, 1985.

[4] G. Wu, S. Bai, and J. Kepler. Mobile platform center shift in spherical parallel manipulators with flexible limbs. Mechanism and Machine Theory, 75:12-26, 2014. 
[5] C.M. Gosselin and J. Angeles. The optimum kinematic design of a spherical three-degree-offreedom parallel manipulator. ASME J. Mech., Trans., and Auto. in Des., 111, 1989.

[6] S. Bai. Optimum design of spherical parallel manipulator for a prescribed workspace. Mech. Mach. Theory, 45(2):200-211, 2010.

[7] C.M. Gosselin and J. Angeles. A global performance index for the kinematic optimization of robotic manipulators. ASME J. Mech. Des., 113(3):220-226, 1991.

[8] S. Bai, M.R. Hansen, and T.O. Andersen. Modelling of a special class of spherical parallel manipulators with Euler parameters. Robotica, 27, 2009.

[9] G. Wu. Multiobjective optimum design of a 3-RRR spherical parallel manipulator with kinematic and dynamic dexterities. Modeling, Identification and Control, 33(3):111-121, 2012.

[10] I.A. Bonev and C.M. Gosselin. Singularity loci of spherical parallel mechanisms. In Proceedings of the 2005 IEEE International Conference on Robotics and Automation, pages 2957-2962, April 2005.

[11] F. Bidault, C.-P. Teng, and J. Angeles. Structural optimization of a spherical parallel manipulator using a two-level approach. In ASME Design Engineering Technical Conferences and Computers and Information in Engineering Conference, Pittsburgh, Pennsylvania, 2001.

[12] S. Staicu. Recursive modelling in dynamics of Agile Wrist spherical parallel robot. Robotics and Computer-Integrated Manufacturing, 25(2):409-416, 2009.

[13] G. Wu, S. Caro, S. Bai, and J. Kepler. Dynamic modeling and design optimization of a 3-DOF spherical parallel manipulator. Robotics and Autonomous Systems, 62:1377-1386, 2014.

[14] H. Alt. Der Üertragungswinkel und seine bedeutung für das konstruieren periodischer getriebe. Werkstattstechnik, 26:S. 61/64, 1932.

[15] K. Hain. Applied Kinematics. McGraw-Hill, 1967.

[16] T.L. Dresner and K.W. Buffinton. Definition of pressure and transmission angles applicable to multi-input mechanisms. ASME Journal of Mechanical Design, 113:495-499, 1991.

[17] S. Bawab, G.L. Kinzel, and K.J. Waldron. Rectified synthesis of six-bar mechanisms with welldefined transmission angles for four-position motion generation. ASME Journal of Mechanical Design, 118:377-383, 1996.

[18] Y. Takeda and H. Funabashi. A transmission index for in-parallel wire-driven mechanisms. JSME International Journal Series C: Mechanical Systems, Machine Elements and Manufacturing, 44(1):180-187, 2001.

[19] R.S. Ball. A Treatise on the Theory of Screws. Cambridge University Press, 1900. 
[20] M.S.C. Yuan, F. Freudenstwin, and L.S. Woo. Kinematic analysis of spatial mechanism by means of screw coordinates. part 2-analysis of spatial mechanisms. Journal of Manufacturing Science and Engineering, 93(1):67-73, 1971.

[21] G. Sutherland and B. Roth. A transmission index for spatial mechanisms. ASME Journal of Manufacturing Science and Engineering, 95(2):589-597, 1973.

[22] C. Chen and J. Angeles. Generalized transmission index and transmission quality for spatial linkages. Mechanism and Machine Theory, 42(9):1225-1237, 2007.

[23] C. Wu, X.-J. Liu, L. Wang, and J. Wang. Optimal design of spherical 5R parallel manipulators considering the motion/force transmissibility. ASME Journal of Mechanical Design, 132(3):031002, 2010.

[24] J. Wang, C. Wu, and X.-J. Liu. Performance evaluation of parallel manipulators: Motion/force transmissibility and its index. Mechanism and Machine Theory, 45(10):1462-1476, 2010.

[25] X. J. Liu, C. Wu, and J. Wang. A new approach for singularity analysis and closeness measurement to singularities of parallel manipulators. Journal of Mechanisms and Robotics, 4(4):041001, 2012.

[26] H. Liu, T. Huang, A. Kecskeméthy, and D. G. Chetwynd. A generalized approach for computing the transmission index of parallel mechanisms. Mechanism and Machine Theory, 74:245-256, 2014.

[27] J. Angeles. Fundamentals of Robotic Mechanical Systems: Theory, Methods, and Algorithms. Springer, NY, USA, 2007.

[28] J. Weisbach and G. Herrmann. Mechanics of Engineering and of Machinery, volume III. Wiley, 1883.

[29] A. Yang and F. Freudenstein. Application of dual-number quaternion algebra to the analysis of spatial mechanisms. ASME Journal of Applied Mechanics, 31:300-307, 1964.

[30] S. Bai and J. Angeles. A unified input-output analysis of four-bar linkages. Mechanism and Machine Theory, 43(2):240-251, 2008.

[31] R. Hartenberg and J. Denavit. Kinematic Synthesis of Linkages. McGraw-Hill, New York, 1964.

[32] D.C. Tao. Applied Linkage Synthesis. Addison-Wesley, Reading, MA, 1964. 